\title{
Will 14-3-3 $\eta$ Be a New Diagnostic and Prognostic Biomarker in Rheumatoid Arthritis? A Prospective Study of Its Utility in Early Diagnosis and Response to Treatment
}

\author{
Doaa Shawky Alashkar, ${ }^{1}$ Radwa Mostafa Elkhouly ${ }^{(D},{ }^{1}$ Amira Yousef Abd Elnaby, ${ }^{2}$ \\ and Doaa Waseem Nada ${ }^{1}$ \\ ${ }^{1}$ Physical Medicine, Rheumatology and Rehabilitation, Faculty of Medicine, Tanta University, Tanta, Egypt \\ ${ }^{2}$ Clinical Pathology, Faculty of Medicine, Tanta University, Tanta, Egypt \\ Correspondence should be addressed to Radwa Mostafa Elkhouly; r_elkhouly@yahoo.com
}

Received 2 October 2021; Accepted 11 December 2021; Published 4 January 2022

Academic Editor: Rizgar Mageed

Copyright (C) 2022 Doaa Shawky Alashkar et al. This is an open access article distributed under the Creative Commons Attribution License, which permits unrestricted use, distribution, and reproduction in any medium, provided the original work is properly cited.

\begin{abstract}
Aim of the Work. To evaluate diagnostic and prognostic capacity of 14-3-3 $\eta$ protein in early RA, investigate its pathogenic and theragnostic role, and find its correlations with disease activity and severity in established RA patients. Subjects and Methods: 80 patients with early RA, 80 patients with established RA, and 80 healthy controls were included in this study. ROC curve analysis of RF, ACCP, and 14-3-3 $\eta$ in early disease was conducted, and serum levels of 14-3-3 $\eta$ were assessed by ELISA and reassessed in early RA patients 6 months after anti-TNF therapy. Correlation of 14-3-3 $\eta$ with parameters of disease activity and severity was analyzed. Results. Serum 14-3-3 $\eta$ levels were significantly higher in all RA patients than in controls $(P<0.001)$, its sensitivity was $86.7 \%$ and $88.3 \%$ in early and established RA patients with a significant difference with RF and ACCP at early disease, and the specificity was $96.7 \%$. There was a significant reduction of $14-3-3 \eta$ levels 6 months after treatment in the first group $(p=0.004)$, and there was a significant positive correlation between serum 14-3-3 $\eta$ levels and parameters of disease activity and severity. Conclusion. 14-3-3 $\eta$ could be a novel, potent, and efficacious diagnostic, and prognostic marker for RA with high sensitivity, that may become a new therapeutic target for RA.
\end{abstract}

\section{Introduction}

Rheumatoid arthritis (RA) is a chronic autoimmune disease that affects about $1.5 \%$ of the population. There are multiple pathophysiological factors in its etiology and manifestations, and there is high heterogeneity among patients throughout the disease. If untreated, RA results in severe joint destruction, leading to impaired physical activity and disability [1]. It is now understood that the disease outcome and patient prognosis can be significantly improved by early identification of RA and prediction of the disease severity at diagnosis for the implementation of an effective treatment strategy [2].

However, early diagnosis of RA is difficult due to limited clinical symptoms and an absence of physical signs.
Laboratory data of patients with arthralgia, such as levels of rheumatoid factor (RF) and anticyclic citrullinated peptide (ACCP), are not remarkable for RA. Therefore, there is a great need for the development and validation of new biomarkers that have predictive capacity for RA [3].

$\mathrm{RF}$ is an autoantibody that targets the Fc region of IgG. The sensitivity of RF testing is 60 to $86 \%$ for established RA and $57 \%$ for early RA, but the utility of RF testing is somewhat limited by its low specificity of 70 to $85 \%$ for established and early RA [4]. Recently, in 2010, cyclic citrullinated peptide (CCP) was added to the classification criteria for RA of the American College of Rheumatology/ European League Against Rheumatism (ACR/EULAR) [5]. CCP testing has sensitivity similar to that of RF for established RA (64\% to 88\%) and early RA (59\%) [6], but its 
specificity (90 to $99 \%$ ) is higher for both established and early disease [7].

Although CCP is more specific than RF testing, it is not considered a replacement for RF. Serology tests for both markers are included in the ACR/EULAR classification criteria for RA [5], and studies show that the combined use of the markers provides greater sensitivity. However, $28-44 \%$ of patients with early RA experience negative test results, and patients who develop erosive RA may remain negative for both markers. Thus, other markers for RA have been investigated [8].

14-3-3 proteins represent a family of intracellular chaperonins that are exclusively expressed in eukaryotic cells. Seven isoforms that share more than $50 \%$ amino acid homology have been isolated: beta $(\beta)$, epsilon $(\varepsilon)$, gamma $(\gamma)$, eta $(\eta)$, tau $(\tau)$, zeta $(\zeta)$, and sigma $(\sigma)$. The $14-3-3$ family can interact with more than 200 intracellular proteins [9]. Thus, it is involved in coordinating an array of biological processes, including protein trafficking, signaling, and cytoskeletal transport [10].

14-3-3 $\eta$ protein represents a novel biomarker for the detection of RA. It may play a role in stimulating tumor necrosis factor-alpha, metalloproteinases, and other inflammatory mediators that are critical to joint erosion. This demonstrates its prognostic value with aims for treatment. Blood levels of 14-3-3 $\eta$ tend to be elevated in patients with RA, but not in those with other collagen diseases [11]. One of the advantages of 14-3-3 $\eta$ as an RA marker is that it can improve the identification rates of early RA. Maksymowych et al. found that adding 14-3-3 $\eta$ to RF and CCP antibody testing increased diagnostic sensitivity for early RA patients [11].

This study aims to evaluate the role of $14-3-3 \eta$ in the early diagnosis of RA, as well as its sensitivity and specificity relative to traditional biomarkers. Furthermore, we defined the correlation of 14-3-3 $\eta$ with the disease's activity, severity, and ultrasonographic features from RA patients with established disease. Lastly, we clarify the effect of anti-TNF therapy (etanercept) on 14-3-3 $\eta$ levels in early RA patients.

\section{Subjects and Method}

Study population: 80 patients early diagnosed to have RA (group I), 80 patients with established disease (group II), and 80 age- and sex-matched healthy volunteers (group III) were included in the study. All patients were selected from Physical Medicine, Rheumatology, and Rehabilitation Clinics, Tanta University Hospitals. The ethical approval was obtained from the hospital ethical research committee of the Faculty of Medicine at Tanta University, approval code: $32768 / 12 / 20$. All investigations were explained to all patients, each patient entering the study was informed and gave written informed consent, and the trial was conducted according to the Declaration of Helsinki principles.

RA patients were diagnosed according to the American College of Rheumatology (ACR)/European League Against Rheumatism (EULAR) 2010 Criteria for RA [5], and patients with other autoimmune diseases, infections, osteoporosis, and malignancy were excluded.

\subsection{Methods}

2.1.1. Group I (Early RA $\leq 6$ Months). Laboratory assessment

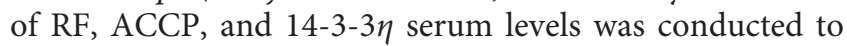
calculate the sensitivity and specificity of each at early disease onset. Serum levels of 14-3-3 $\eta$ were reassessed 6 months after anti-TNF therapy (etanercept; Enbrel with a dose of $50 \mathrm{mg} /$ week SC).

Laboratory assessment of serum 14-3-3 $\eta$ level: venous blood samples were collected and centrifuged at $1000 \mathrm{~g}$ for $15 \mathrm{~min}$ for serum separation. The serum samples were frozen at $-70^{\circ} \mathrm{C}$ until used for assay of serum 14-3-3 $\eta$ level using the Human 14-3-3 Protein (14-3-3 Pro) ELISA Kit Cat No. MBS269690 using the double-sandwich ELISA technique. The precoated antibody is a human 14-3-3 promonoclonal antibody, and the detecting antibody is a polyclonal antibody with biotin labeled. Samples and biotin labeling antibodies are added into ELISA plate wells and washed out with PBS or TBS. Then Avidin-peroxidase conjugates are added to ELISA wells in order; TMB substrate was used for coloring after the reactant is thoroughly washed out by PBS or TBS. TMB turns into blue in peroxidase catalytic and finally turns into yellow under the action of acid. The color depth and the testing factors in samples are positively correlated [12].

2.1.2. Group II: Clinical and Laboratory Assessment. Full medical history taking and general and locomotor system examination: assessment of activity by Disease Activity Score 28 (DAS-28):

DAS-28 $=0.56 \sqrt{ }($ TEN28 $)+0.28 \sqrt{ }($ SW 28$)+0.70 \mathrm{Ln}$ $(\mathrm{ESR})+0.014$ [13]. Assessment of severity by the rheumatoid arthritis severity scale (RASS) that consists of three scales (disease activity, functional impairment, and physical damage) was conducted using a range of 1-100, with a score of 1 meaning there is no evidence of the condition, and 100 means maximum level of progression [14]. Serum levels of RF, ACCP, and 14-3-3 $\eta$ were assessed to determine their sensitivity and specificity in patients with established disease to be compared with those in patients at early disease onset and laboratory assessment of ESR and CRP.

Radiographic assessment: plain X-ray of both hands and wrists was performed, sixteen joints were evaluated in each hand using the modified Larsen score MLS 1995, and the final score ranges from 0 to 160 [15].

Ultrasonographic assessment: systematic multiplanar gray-scale ultrasound (GSUS) and power Doppler ultrasound (PDUS) examinations were performed on the most clinically affected wrist joint, in a standardized manner based on the guidelines of the European League Against Rheumatism (EULAR) using joint inflammation and damage parameters (synovial thickening, joint effusion, bone erosion, and power Doppler PD activity): (1) synovial thickening: nondisplaceable and poorly compressible visualized abnormal hypoechoic intra-articular tissue, in longitudinal and transverse planes, measured in millimeters, 2) joint effusion: a compressible anechoic intracapsular area that is semiquantitatively examined as follows: grade 0: no 
effusion; grade 1: minimal; grade 2: moderate (without distension of the joint capsule); and grade 3: extensive (with distension of the joint capsule), 3) vascularity by Power Doppler (PD): semiquantitative grading of the PD was assessed as follows: grade 0: no flow in the synovium; grade 1: single vessel signal; grade 2: less than half of the area of the synovium is filled with vessel signal; and grade 3: more than half of the area is filled with vessel signal, and 4) bone erosion score: an interruption of the bone surface on two perpendicular planes defined as erosion, assessed as follows: grade 0: normal bone surface; grade 1: bone surface irregularity without defect in two planes; grade 2: surface defect in two planes; and grade 3: bone defect creating extensive bone destruction [16, 17].

Statistical analysis was carried out using the statistical package for social sciences (SPSS) software, version 16.0 for Windows (SPSS Inc., Chicago, Illinois, USA). Demographic data between patients and controls were compared using chi-square and unpaired Student's $t$-tests. Pearson's correlation coefficient $(r)$ was used to determine the correlations. Data are expressed as a mean \pm SD. $P$ values of less than 0.05 were considered statistically significant and less than 0.001 were considered highly significant for differences and correlations.

\section{Results}

The results of this study revealed a highly significant difference between 14-3-3 $\eta$, RF, and ACCP regarding sensitivity for RA diagnosis at early disease onset $(p<0.001)$, but not in established disease. The sensitivity of $14-3-3 \eta$ was $86.7 \%$ and $88.3 \%$ in early and established RA, respectively. Serum levels of $14-3-3 \eta$ were highly significant in all RA patients compared to controls $(p<0.001)$, and there was a significant reduction at 6 months after anti-TNF therapy in patients with early disease $(p=0.004)$ (Table 1$)$.

Regarding parameters of active disease in patients with established RA, the median DAS was 4.13 , and the synovial thickness of the most affected wrist was $2.85 \mathrm{~mm}$ according to ultrasound. Most patients showed grade 2 joint effusion and Doppler activity. Assessment of disease severity in these patients revealed a median of Modified Larsen Score (MLS) of 34 and RASS of 70 , with $31.7 \%$ showing grade 2 bone erosions in $31.7 \%$ of them (Figure 1 and Table 2).

14-3-3 $\eta$ showed a significant positive correlation with clinical, laboratory, and radiological parameters of disease activity and severity in patients with established disease (Table 3). ROC curve analysis yielded sensitivities of $61.7 \%$, $68.3 \%$, and $86.7 \%$ for RF, ACCP, and $14-3-3 \eta$ at early disease onset at cutoff values of $7.93,21.20$, and 0.23 with a specificity of $88.3 \%, 95 \%$, and $96.7 \%$, respectively (Figure 2 and Table 4).

\section{Discussion}

Early diagnosis of RA is now a matter of interest to prevent joint damage and improve clinical and functional outcomes. Seronegative of RF and ACCP at early disease stages necessitates a critical need to identify new biomarkers with high diagnostic potential at early disease onset $[18,19]$.

Seven isoforms of the 14-3-3 protein family have been isolated, and one of these is the 14-3-3 $\eta$ protein. This isoform is highly expressed extracellularly in the joints of patients with erosive RA and strongly correlated with MMP-1 and MMP3 in both synovial fluid and serum. This characterizes its biological expression and pathogenic role in rheumatologic disease processes [20]. A study of synovial fluid and serum from patients with inflammatory arthritis revealed the presence of two isoforms of 14-3-3, $\eta$ and $\eta$, with predominance of the latter isoform based on mass spectrometry data [21].

14-3-3 proteins have been described as a key component of exosomes, so their extracellular expression in RA is believed to be mediated in part through an exosomal process. Externalization of 14-3-3 proteins occurs as a result of an active secretory mechanism in inflammation, which is suggested by their release from activated immune cells, including dendritic cells, $T$ cells, $B$ cells, macrophages, and epithelial cells [22]. Thus, this study was designed to evaluate the diagnostic utility of 14-3-3 $\eta$ protein in patients with early RA compared to the traditional biomarkers RF and ACCP, its role in disease pathogenesis, and hence, its theragnostic utility. In addition, we evaluated its prognostic role in disease progression in patients with established RA.

The sensitivities of RF in patients with early and established RA were $61.7 \%$ and $78.3 \%$, respectively, and the difference between them was significant. Furthermore, there was a significant difference between the sensitivity of ACCP between the two groups (68.3-85\%). In contrast, the difference between $14-3-3 \eta$ in patients of both groups was insignificant (86.7-88.3\%). The difference between 14-3-3 $\eta$, $\mathrm{RF}$, and ACCP was highly significant in patients with early RA, indicating its high diagnostic utility at early disease stages compared to other biomarkers.

At early disease stages, the specificities of RF, ACCP, and 14-3-3 $\eta$ were $88.3 \%$, 95\%, and 96.7\%, respectively. Furthermore, our results revealed that $36.7 \%$ of patients in the first group with negative RF results and $28.8 \%$ with negative ACCP results were positive for $14-3-3 \eta$. This finding draws attention to the value of $14-3-3 \eta$ in the early identification of RA.

ROC curve analysis for RF, ACCP, and $14-3-3 \eta$ at early disease onset yielded a significant difference in the results regarding sensitivity with cutoff values of $>7.93,>21.20$, and $>0.234$, respectively. The diagnostic utility of $14-3-3 \eta$ in early disease onset for effective treatment strategies and better outcomes in RA was also assessed by Maksymowych et al. in patients with a median disease duration of $<3.5$ months. At a cutoff of $\geq 0.19 \mathrm{ng} / \mathrm{mL}$, the ROC curve yielded a sensitivity of $63.6 \%$ and a specificity of $92.6 \%$. The incremental benefit of adding $14-3-3 \eta$ to ACCP and RF resulted in an identification rate of $72 \%$ compared to $59 \%$ for ACCP alone, and diagnostic capture was increased from $59-72 \%$ to $78 \%$ when 14 $3-3 \eta$ was added to RF [11].

In a study conducted on 619 subjects, the ROC curve analysis demonstrated a significant area under the curve 
TABLE 1: A comparison of the sensitivity of various RA biomarkers in patients with early and established disease. Serum 14-3-3 levels (ng/ml) were compared between the two RA groups, as well as the control and comparison of serum 14-3-3 levels before and after anti-TNF therapy in group I patients.

\begin{tabular}{|c|c|c|c|c|}
\hline & Group I (early RA) $(n=80)$ & Group II (established RA) $(n=80)$ & $\chi^{2}:$ & ${ }^{\mathrm{MC}} p$ \\
\hline $\mathrm{RF}$ & $61.7 \%$ & $78.3 \%$ & 3.968 & $0.0464^{*}$ \\
\hline ACCP & $68.3 \%$ & $85 \%$ & 4.658 & $0.0309^{*}$ \\
\hline $14-3-3 \eta$ & $86.7 \%$ & $88.3 \%$ & 0.0762 & 0.0783 \\
\hline$\chi^{2}:{ }^{\mathrm{MC}} p$ & $17.023<0.001^{*}$ & 3.8540 .146 & & \\
\hline 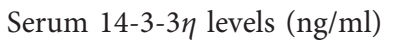 & Group I (early RA) $(n=80)$ & Control & $t$ & $P$ \\
\hline Min.-Max. & $0.15-4.3$ & $0.00-1.26$ & 25.45 & $<0.001^{*}$ \\
\hline Mean \pm SD & $2.34 \pm 0.42$ & $0.13 \pm 0.56$ & & \\
\hline Serum $14-3-3 \eta$ levels & Group II (established RA) $(n=80)$ & Control & $t$ & $P$ \\
\hline Min.-Max. & $0.17-5.1$ & $0.00-1.26$ & 37.88 & $<0.001^{*}$ \\
\hline Mean \pm SD & $3.15 \pm 0.26$ & $0.13 \pm 0.56$ & & \\
\hline Serum $14-3-3 \eta$ in group I & Mean \pm SD & \multicolumn{2}{|l|}{$T$} & $p$ \\
\hline Before treatment & $2.34 \pm 0.42$ & \multirow{2}{*}{\multicolumn{2}{|c|}{2.952}} & $0.004^{*}$ \\
\hline After treatment & $2.03 \pm 0.63$ & & & \\
\hline
\end{tabular}

MC: Monte Carlo; $\chi^{2}$ : chi-square test.

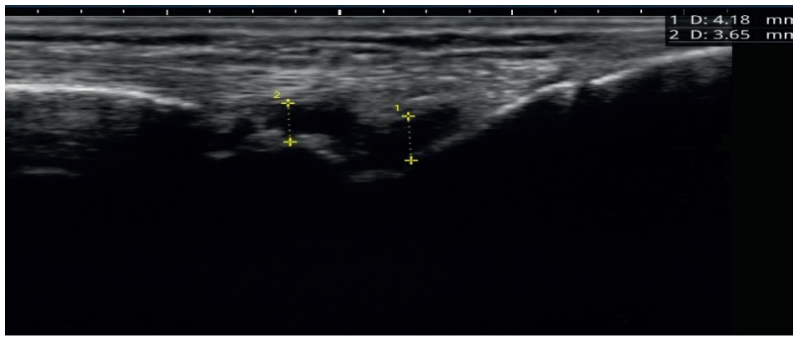

(a)

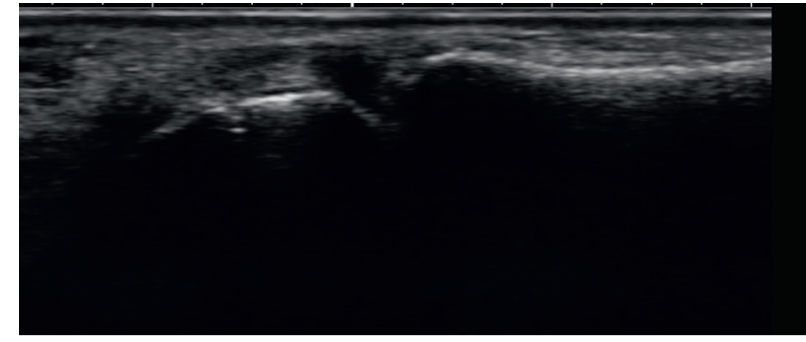

(b)

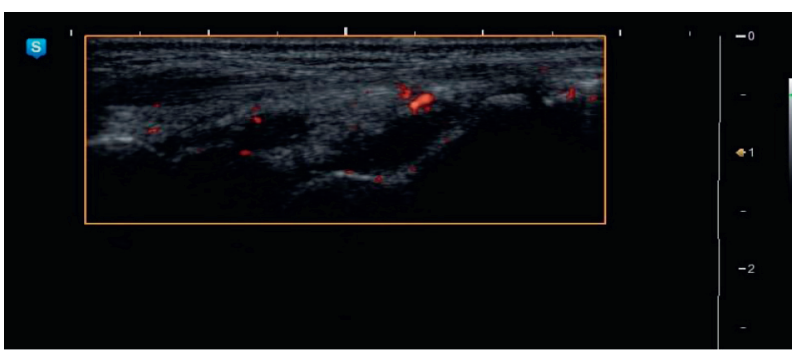

(c)

FIgURE 1: (a) Ultrasonographic examination of the wrist of an RA patient showing synovial thickness: at radiocarpal $=4.18 \mathrm{~mm}$ and intercarpal $=3.65 \mathrm{~mm}$, (b) ultrasonographic examination of the wrist of an RA patient showing grade 2 carpal bone erosion, and (c) ultrasonographic examination of the wrist of an RA patient showing grade 2 Doppler activity.

(AUC) of 0.89 with a cutoff of $\geq 0.19 \mathrm{ng} / \mathrm{mL}$, yielding $77.0 \%$ sensitivity and $92.6 \%$ specificity [23].

In a study by Maksymowych et al., the ROC curve of 143- $3 \eta$ autoantibodies for early RA versus healthy and diseaserelevant controls yielded a sensitivity of $73 \%$ and a specificity of $79 \%$. Unlike $14-3-3 \eta$ protein, no significant correlation was observed with RF or ACPA. Also, no significant correlation was observed between titers of $14-3-3 \eta$ protein and its autoantibodies. However, their combined expression identified more than $90 \%$ of early RA patients [24]. While

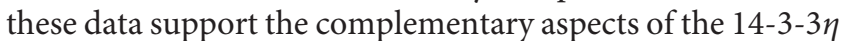
biomarker platform, further validation according to the
OMERACT framework is necessary before $14-3-3 \eta$ biomarkers attain the status of a monitoring marker [24].

In the current study, serum levels of $14-3-3 \eta$ in patients of both RA groups were significantly higher compared to controls. Similarly, the median 14-3-3 $\eta$ levels in early RA patients were significantly higher than in disease-relevant controls ( 0.76 vs. $0.02 \mathrm{ng} / \mathrm{mL}$ ) in the study by Maksymowych et al. [11]. These levels were three to five times higher than the corresponding levels in the serum of matched donors [21].

In another study, 619 subjects including healthy and disease controls (with other arthropathies, connective tissue 
TABLE 2: Clinical and radiological parameters of disease activity and clinical and radiological parameters of disease severity in patients with established RA.

\begin{tabular}{|c|c|c|c|}
\hline Disease severity & Min.-Max. & Mean \pm SD & Median \\
\hline RASS & $40.0-100.0$ & $77.50 \pm 18.03$ & 70.0 \\
\hline Modified Larsen score MLS & $20.0-64.0$ & $34.30 \pm 14.06$ & 34.0 \\
\hline Disease activity score (DAS 28) & $2.65-5.24$ & $3.93 \pm 1.35$ & 4.13 \\
\hline Ultrasonographic assessment (syn & & & \\
\hline Min.-Max. & & \multirow{3}{*}{\multicolumn{2}{|c|}{$\begin{array}{c}1.95 \mathrm{~mm}-4.80 \mathrm{~mm} \\
3.12 \pm 1.24 \mathrm{~mm} \\
2.85 \mathrm{~mm}\end{array}$}} \\
\hline Mean \pm SD & & & \\
\hline Median & & & \\
\hline Joint effusion & & No. & $\%$ \\
\hline Grade 0 & & 9 & 15 \\
\hline Grade 1 & & 17 & 28.3 \\
\hline Grade 2 & & 18 & 30.0 \\
\hline Grade 3 & & 16 & 26.7 \\
\hline \multicolumn{4}{|l|}{ Doppler activity } \\
\hline Grade 0 & 12 & 20.0 & \\
\hline Grade 1 & 16 & 26.7 & \\
\hline Grade 2 & 22 & 36.6 & \\
\hline Grade 3 & 10 & 16.7 & \\
\hline Bone erosion (by US) & No. & \multicolumn{2}{|c|}{$\%$} \\
\hline Grade 0 & 9 & \multicolumn{2}{|c|}{15.0} \\
\hline Grade 1 & 14 & \multicolumn{2}{|c|}{23.3} \\
\hline Grade 2 & 19 & \multicolumn{2}{|c|}{31.7} \\
\hline Grade 3 & 18 & \multicolumn{2}{|c|}{30.0} \\
\hline
\end{tabular}

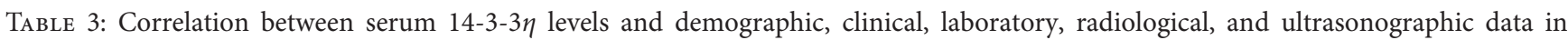
established RA patients.

\begin{tabular}{lccc}
\hline & $r_{s}$ & $H$ & \multicolumn{1}{c}{$P$} \\
\hline DAS 28 score & 0.837 & & $<0.001^{*}$ \\
ESR (mm/h) & $\mathbf{0 . 9 0 8}$ & & $\mathbf{0 . 0 0 1}^{*}$ \\
CRP (mg/dl) & $\mathbf{0 . 3 8 2}$ & & $\mathbf{0 . 0 4 5}^{*}$ \\
RF (IU) & $\mathbf{0 . 7 6 7}$ & & $\mathbf{0 . 0 0 9}^{*}$ \\
ACCP (IU) & $\mathbf{0 . 6 6 7}$ & & $\mathbf{0 . 0 0 1}^{*}$ \\
RASS & $\mathbf{0 . 7 0 6}$ & & $\mathbf{0 . 0 4 2}^{*}$ \\
Modified Larsen score & $\mathbf{0 . 4 3 5}$ & & $\mathbf{0 . 0 3 3}^{*}$ \\
Synovial thickness & $\mathbf{0 . 6 7 6}$ & $\mathbf{H}=\mathbf{3 . 3 4 1}^{*}$ & $\mathbf{0 . 0 0 1}^{*}$ \\
Effusion & & $\mathbf{H}=\mathbf{4 . 5 1 7}^{*}$ & $\mathbf{0 . 0 1 7}^{*}$ \\
Erosions & & $\mathbf{H}=\mathbf{8 . 7 5 6}^{*}$ & $\mathbf{0 . 0 2 3}^{*}$ \\
Doppler signal & & $\mathbf{0 . 0 4 8}^{*}$ \\
\hline
\end{tabular}

H: Kruskal-Wallis test.

disorders, and autoimmune diseases) were assessed for se-

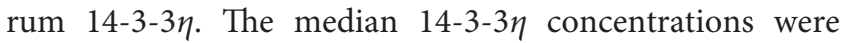
significantly higher in established RA patients versus healthy individuals (1.12 vs. $0.00 \mathrm{ng} / \mathrm{mL})$ and controls $(0.02 \mathrm{ng} / \mathrm{mL})$ [22]. Regarding the diagnostic utility of $14-3-3 \eta$ in undifferentiated RA, 148 patients with arthralgia were recruited in a cohort study to determine whether it was associated with the development of RA. The results revealed that the median 14-3-3 $\eta$ levels were significantly higher in the arthralgia group that developed RA, while the 14-3-3 $\eta$ and ACCP titers (but not titers of RF) were associated with the development of RA with LRs of 4.12 and 4.16 [23].

To determine the pathogenic role of $14-3-3 \eta$, in vitro cell stimulation studies were performed using human recombinant $14-3-3 \eta$ at concentrations similar to that found in RA patients' sera. The studies revealed that 14-3-3n preferentially stimulates cells of the innate immune system, leading to activation of key signaling cascades such as the MAPK/ERK, SAPK/JNK, and the JAK-STAT pathway, which regulate the production of inflammatory and degradative factors responsible for joint damage. Furthermore, several RA-relevant transcripts were upregulated by 14-3-3 $\eta$, including proinflammatory cytokines interleukin (IL) $-1 \beta$, IL-6, and TNF- $\alpha$, as well as joint degradation factors such as MMP-9 and receptor activator of nuclear factor kappa-B ligand (RANKL). These factors can be blocked by preincubation with 14-3-3 $\eta$ antibodies [22].

At 6 months after anti-TNF therapy, there was a significant reduction of serum $14-3-3 \eta$ levels in patients with early disease onset, suggesting a role in disease 


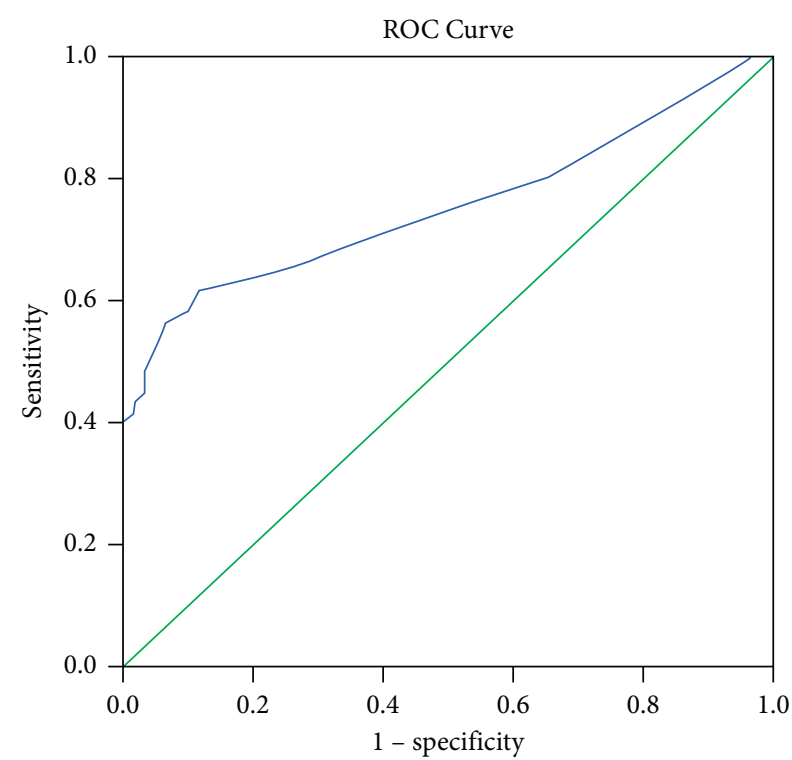

(a)

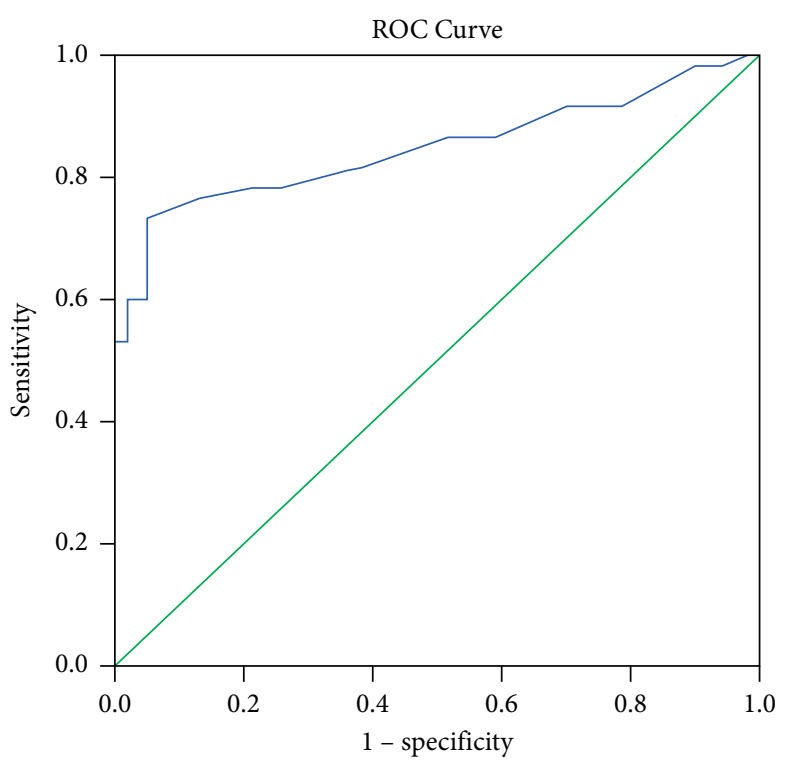

(b)

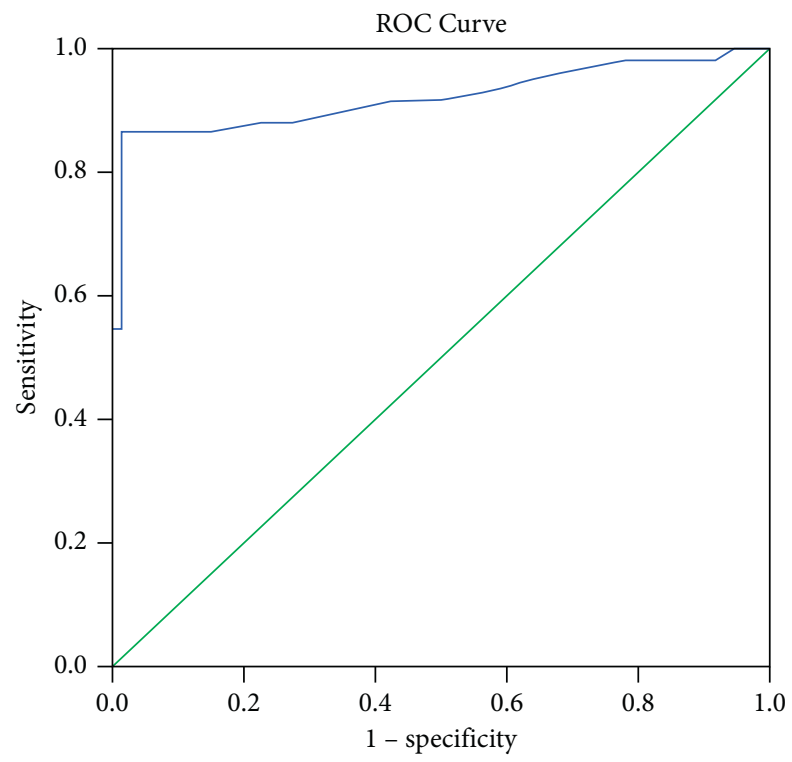

(c)

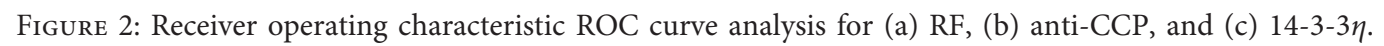

TABle 4: Agreement (sensitivity and specificity) for serum RF, ACCP, and 14-3-3 $\eta$ to predict early RA patients (vs. control).

\begin{tabular}{|c|c|c|c|c|c|c|c|c|c|}
\hline & \multirow[b]{2}{*}{ AUC } & \multirow[b]{2}{*}{$p$} & \multicolumn{2}{|c|}{$95 \%$ CI } & \multirow[b]{2}{*}{ Cutoff } & \multirow[b]{2}{*}{ Sensitivity } & \multirow[b]{2}{*}{ Specificity } & \multirow[b]{2}{*}{ PPV } & \multirow[b]{2}{*}{ NPV } \\
\hline & & & LL & UL & & & & & \\
\hline $\mathrm{RF}$ & 0.750 & $<0.001^{*}$ & 0.646 & 0.830 & $>7.93$ & 61.7 & 88.3 & 84.0 & 69.7 \\
\hline ACCP & 0.817 & $<0.001^{*}$ & 0.732 & 0.913 & $>21.20$ & 68.3 & 95.0 & 93.1 & 75.0 \\
\hline $14-3-3 \eta$ & 0.917 & $<0.001^{*}$ & 0.854 & 0.998 & $>0.234$ & 86.7 & 96.7 & 96.3 & 87.9 \\
\hline
\end{tabular}

AUC: area under a curve, $p$ value: probability value, CI: confidence interval, NPV: negative predictive value, PPV: positive predictive value, ${ }^{*}$ : statistically significant at $p \leq 0.05$

pathogenesis and potential as a therapeutic target for disease remission. Efforts are underway to understand the interplay between $14-3-3 \eta$ and various chemokines/cytokines relevant to RA, as well as how specific therapies impact 14-3-3 $\eta$ serum levels [24]. Furthermore, studies are currently being conducted to investigate the therapeutic potential of targeting $14-3-3 \eta$ in vivo using a collageninduced arthritis model [22]. 
Regarding patients with established RA (group 2), disease activity was assessed clinically by DAS-28 (median 4.13) and ultrasonographic findings. Current data indicate a good correlation of MSUS with classical measures of clinical activity, and in some instances, MSUS appears to perform even better and has priority for the diagnosis of subclinical synovitis, which might help physicians in treatment planning [25]. The median synovial thickness in RA patients was $2.85 \mathrm{~mm}, 30 \%$ of patients showed grade 2 effusion, and $36.6 \%$ presented grade 2 Doppler activity.

We observed a highly significant positive correlation between serum 14-3-3 $\eta$ levels and DAS-28 and ultrasonographic findings in our patients of established disease $(p<0.001)$. Our study showed a significant positive correlation of serum 14-3-3 $\eta$ levels with ESR and CRP ( $p<0.001$ and 0.045 , respectively). High CRP levels lead to the consideration that a progressive and erosive disease is present, so elevated serum 14-3-3 $\eta$ levels indicate disease

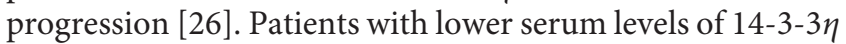
at disease onset showed better clinical outcomes and a higher likelihood of achieving DAS remission in response to standard DMARDs. This was explained by an interplay that exists between 14-3-3 $\eta$ and TNF- $\alpha$. More specifically, 14-3$3 \eta$ can induce various inflammatory factors (including IL-6) in a dose-dependent fashion and may perpetuate the "cytokine storm" [24-27].

The median of RASS among RA patients was 70, and it was significantly correlated with $14-3-3 \eta$ serum levels. The median MLS in RA patients was 34 , and $31.7 \%$ of RA patients showed grade 2 bone erosion in ultrasonographic examination. Also, there was a significant positive correlation between 14-3-3 $\eta$ serum levels and radiological indicators of joint damage and disease severity.

14-3-3 $\eta$ is a potent, dose-dependent upregulation factor that perpetuates joint damage. Preliminary data from an analysis of 33 patients with RA revealed that median 14-3-3n levels were significantly higher in early RA patients with radiographic progression versus those of patients who did not progress $(2.68 \mathrm{ng} / \mathrm{ml}$ vs. $0.09 \mathrm{ng} / \mathrm{ml})$ [28]. A previous cohort study was conducted to demonstrate its role in disease prognosis, and 409 patients with early RA were recruited. Radiographic progression was assessed by the Sharp-van der Heijde score (SHS). 67\% of patients were 14$3-3 \eta$ positive, and in patients who progressed radiographically by year 5, there were significantly higher levels of 14-3$3 \eta$ and RF, but not ACPA. Positive 14-3-3 $\eta$ status was significantly associated with radiographic progression at years 1, 3, and 5 with LR ranging from 3.8 to 6.8 [29].

Hirata et al. also reported this positive correlation, and the higher the 14-3-3 $\eta$ baseline levels were, the stronger the association with radiographic progression was [30]. Another study revealed significantly higher levels of $14-3-3 \eta$ in patients who already had radiographic joint damage at the study baseline, as well as in those who developed progression by the end of the follow-up period. Monocytes from the THP-1 cell line were stimulated with $14-3-3 \eta$ in a concentration similar to that of those found in the sera of RA patients. This resulted in the induction of proinflammatory cytokines IL- $1 \beta$, IL-6, and TNF- $\alpha$, as well as joint degradation factors such as MMP-
9 and RANKL [22]. There was a significant positive correlation of $14-3-3 \eta$ with RF and ACPA.

Forslund et al. [31] demonstrated the predictive value of ACPA for radiological changes. Other studies investigated that the ACCP antibody is better as a predictor of the disease course over 3 years $[32,33]$. This supports the results of our study that the presence of ACCP and serum 14-3-3 $\eta$ is associated with joint destruction. Nevertheless, several independent multicenter studies are required to expand our understanding of the diagnostic, prognostic, and theragnostic applications of 14-3-3 $\eta$ biomarkers in RA for early therapeutic interventions and improving disease outcomes.

\section{Conclusions}

Conclusions: $14-3-3 \eta$ has potential utility in the early diagnosis of RA with higher sensitivity and specificity compared to traditional diagnostic biomarkers. The addition of 14-3-3 $\eta$ as a novel biomarker to RF and ACCP is beneficial for early diagnosis of RA and early therapeutic intervention to reduce disease progression and structural damage. High serum levels of $14-3-3 \eta$ in RA patients with established disease and its correlation with disease activity and severity reflect its role in disease pathogenesis, as well as its impact on disease progression. Thus, it warrants attention as a target for novel therapies. Furthermore, the significant reduction of serum 14-3-3 $\eta$ levels in patients with early RA at 6 months after anti-TNF therapy draws attention to its role in the pathogenesis and prognosis of the disease.

\section{Data Availability}

The data used to support the findings of this study are included within the article.

\section{Conflicts of Interest}

There are no conflicts of interest concerning this article.

\section{References}

[1] S. M. M. Verstappen, J. W. J. Bijlsma, H. Verkleij et al., "Overview of work disability in rheumatoid arthritis patients as observed in cross-sectional and longitudinal surveys," Arthritis Care \& Research, vol. 51, no. 3, pp. 488-497, 2004.

[2] M. Vermeer, H. H. Kuper, M. Hoekstra et al., "Implementation of a treat-to-target strategy in very early rheumatoid arthritis: results of the dutch rheumatoid arthritis monitoring remission induction cohort study," Arthritis \& Rheumatism, vol. 63, no. 10, pp. 2865-2872, 2011.

[3] M. M. J. Nielen, D. van Schaardenburg, H. W. Reesink et al., "Specific autoantibodies precede the symptoms of rheumatoid arthritis: a study of serial measurements in blood donors," Arthritis \& Rheumatism, vol. 50, no. 2, pp. 380-386, 2004.

[4] A. Greiner, H. Plischke, H. Kellner, and R. Gruber, "Association of anti-cyclic citrullinated peptide antibodies, anticitrullin antibodies, and IgM and IgA rheumatoid factors with serological parameters of disease activity in rheumatoid arthritis," Annals of the New York Academy of Sciences, vol. 1050, no. 1, pp. 295-303, 2005. 
[5] D. Aletaha, T. Neogi, A. J. Silman et al., "2010 Rheumatoid arthritis classification criteria: an American college of rheumatology/European league against rheumatism collaborative initiative," Arthritis \& Rheumatism, vol. 62, no. 9, pp. 2569-2581, 2010.

[6] S. Dubucquoi, E. Solau-Gervais, D. Lefranc et al., "Evaluation of anti-citrullinated filaggrin antibodies as hallmarks for the diagnosis of rheumatic diseases," Annals of the Rheumatic Diseases, vol. 63, no. 4, pp. 415-419, 2004.

[7] D. M. Lee and P. H. Schur, "Clinical utility of the anti-CCP assay in patients with rheumatic diseases," Annals of the Rheumatic Diseases, vol. 62, no. 9, pp. 870-874, 2003.

[8] A. L. Jansen, I. van der Horst-Bruinsma, D. van Schaardenburg, R. J. van de Stadt, M. H. de Koning, and B. A. Dijkmans, "Rheumatoid factor and antibodies to cyclic citrullinated Peptide differentiate rheumatoid arthritis from undifferentiated polyarthritis in patients with early arthritis," Journal of Rheumatology, vol. 29, no. 10, pp. 2074-2076, 2002.

[9] J. Jin, F. D. Smith, C. Stark et al., "Proteomic, functional, and domain-based analysis of in vivo 14-3-3 binding proteins involved in cytoskeletal regulation and cellular organization," Current Biology, vol. 14, no. 16, pp. 1436-1450, 2004.

[10] X. Yang, W. H. Lee, F. Sobott et al., "Structural basis for protein-protein interactions in the 14-3-3 protein family," Proceedings of the National Academy of Sciences, vol. 103, no. 46, pp. 17237-17242, 2006.

[11] W. P. Maksymowych, S. J. Naides, V. Bykerk et al., "Serum 14$3-3 \eta$ is a novel marker that complements current serological measurements to enhance detection of patients with rheumatoid arthritis," Journal of Rheumatology, vol. 41, no. 11, pp. 2104-2113, 2014.

[12] R. M. Lequin, "Enzyme immunoassay (EIA)/enzyme-linked immunosorbent assay (ELISA)," Clinical Chemistry, vol. 51, no. 12, pp. 2415-2418, 2005.

[13] G. Wells, J.-C. Becker, J. Teng et al., "Validation of the 28-joint Disease Activity Score (DAS28) and European League against Rheumatism response criteria based on C-reactive protein against disease progression in patients with rheumatoid arthritis, and comparison with the DAS28 based on erythrocyte sedimentation rate," Annals of the Rheumatic Diseases, vol. 68, no. 6, pp. 954-960, 2009.

[14] W. A. Bardwell, P. M. Nicassio, M. H. Weisman, R. Gevirtz, and D. Bazzo, "Rheumatoid Arthritis Severity Scale: a brief, physician-completed scale not confounded by patient selfreport of psychological functioning," Rheumatology, vol. 41, no. 1, pp. 38-45, 2002.

[15] A. Larsen, "How to apply Larsen score in evaluating radiographs of rheumatoid arthritis in long-term studies," Journal of Rheumatology, vol. 22, no. 10, pp. 1974-1975, 1995.

[16] H. Xiao, M. Liu, L. Tan et al., "Value of ultrasonography for diagnosis of synovitis associated with rheumatoid arthritis," International Journal of Rheumatic Diseases, vol. 17, no. 7, pp. 767-775, 2014.

[17] S. Ohrndorf, A.-M. Glimm, G.-R. Burmester, and M. Backhaus, "Musculoskeletal ultrasound scoring systems: assessing disease activity and therapeutic response in rheumatoid arthritis," International Journal of Clinical Rheumatology, vol. 6, no. 1, pp. 57-65, 2011.

[18] I. B. McInnes and G. Schett, "The pathogenesis of rheumatoid arthritis," New England Journal of Medicine, vol. 365, no. 23, pp. 2205-2219, 2011.
[19] B. Heidari, "Rheumatoid Arthritis: early diagnosis and treatment outcomes," Caspian Journal of internal medicine, vol. 2, no. 1, pp. 161-170, 2011.

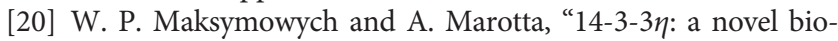
marker platform for rheumatoid arthritis," Clinical \& Experimental Rheumatology, vol. 32, no. 5, pp. S-35-S-39, 2014.

[21] R. T. Kilani, W. P. Maksymowych, A. Aitken et al., "Detection of high levels of 2 specific isoforms of 14-3-3 proteins in synovial fluid from patients with joint inflammation," Journal of Rheumatology, vol. 34, no. 8, pp. 1650-1657, 2007.

[22] C. Théry, M. Ostrowski, and E. Segura, "Membrane vesicles as conveyors of immune responses," Nature Reviews Immunology, vol. 9, no. 8, pp. 581-593, 2009.

[23] W. P. Maksymowych, D. van der Heijde, C. F. Allaart et al., "14-3-3 $\eta$ is a novel mediator associated with the pathogenesis of rheumatoid arthritis and joint damage," Arthritis Research and Therapy, vol. 16, no. 2, pp. 999-R101, 2014.

[24] W. P. Maksymowych, R. Landewé, P.-P. Tak et al., "Reappraisal of OMERACT 8 draft validation criteria for a soluble biomarker reflecting structural damage endpoints in rheumatoid arthritis, psoriatic arthritis, and spondyloarthritis: the OMERACT 9 v2 criteria," Journal of Rheumatology, vol. 36, no. 8, pp. 1785-1791, 2009.

[25] D. Van Schaardenburg, W. P. Maksymowych, M. Boers, M. H. van Beers-Tas, and A. Marotta, "SAT0037 serum 14-33eta predicts the risk of RA development and its higher titres are associated with higher risk," Annals of the Rheumatic Diseases, vol. 73, no. 2, 2014.

[26] E. Naredo, M. A. D’Agostino, R. J. Wakefield, I. Möller, P. V. Balint, and E. Filippucci, "Reliability of a consensusbased ultrasound score for tenosynovitis in rheumatoid arthritis," Annals of the Rheumatic Diseases, vol. 72, no. 8, pp. 1328-1334, 2013.

[27] N. S. Neto and J. F. de Carvalho, "The use of inflammatory laboratory tests in rheumatology," Complement, vol. 3, no. C4, p. C9, 1999.

[28] A. Sagawa, M. Kaneda, Y. Gui, A. Marotta, and J. Arai, "THU0226 evaluation of serum 14-3-3 $\eta$ levels in a Japanese RA cohort treated with tocilizumab," Annals of the Rheumatic Diseases, vol. 73, no. 2, pp. 1-260, 2014.

[29] G. Boire, N. Carrier, A. J. de Brum-Fernandes et al., "SAT0041 14-3-3 $\eta$ predicts radiographic progression in recent-onset polyarthritis patients," Annals of the Rheumatic Diseases, vol. 73, no. 2, pp. 603-604, 2014.

[30] S. Hirata, A. Marotta, Y. Gui, K. Hanami, and Y. Tanaka, "Serum 14-3-3 $\eta$ level is associated with severity and clinical outcomes of rheumatoid arthritis, and its pretreatment level is predictive of DAS28 remission with tocilizumab," Arthritis Research and Therapy, vol. 17, no. 1, pp. 1-0, 2015.

[31] K. Forslind, M. Ahlmén, K. Eberhardt, I. Hafström, and B. Svensson, "Prediction of radiological outcome in early rheumatoid arthritis in clinical practice: role of antibodies to citrullinated peptides (anti-CCP)," Annals of the Rheumatic Diseases, vol. 63, no. 9, pp. 1090-1095, 2004.

[32] A. Kastbom, G. Strandberg, A. Lindroos, and T. Skogh, "AntiCCP antibody test predicts the disease course during 3 years in early rheumatoid arthritis (the Swedish TIRA project)," Annals of the Rheumatic Diseases, vol. 63, no. 9, pp. 1085-1089, 2004.

[33] D. van Schaardenburg, W. P. Maksymowych, M. Boers, S. Turk, and A. Marotta, "THU0244 14-3-3 $\eta$ is an independent predictor of radiographic changes in early RA and higher titres inform a higher likelihood of joint damage progression," Annals of the Rheumatic Diseases, vol. 73, no. 2, pp. 2-266, 2014. 